\title{
Stereo-Microphotography with the Operating Microscope
} A.

Leyden

Author's address:A. Spoor Ph.D., E.N.T. Department, University of Loyden, Leyden (Netherlands)

Stereoscopic pictures taken with the operating microscope have some advantages; the size and extension of a certain part and the relative position of different parts can easily be seen. A special attachment containing a biprism is described. This attachment connected to the tele-objective of a one-eye reflex camera replaces the binocular body of the operating microscope. By a special position of the aperture stop of the camera lens the images of the two built-in objectives of the microscope are separated on the film.

The stereo-slides can be projected by a normal 2" $\times 2$ " projector with a biprism attachment to the lens and polarization filters; a metalized screen and polarizing spectacles must be used. 